Review Article

\title{
The Bidirectional Interactions between Resveratrol and Gut Microbiota: An Insight into Oxidative Stress and Inflammatory Bowel Disease Therapy
}

\author{
Yaolian Hu, ${ }^{1,2,3}$ Daiwen Chen, ${ }^{1,2,3}$ Ping Zheng, ${ }^{1,2,3}$ Jie Yu, ${ }^{1,2,3}$ Jun He, ${ }^{1,2,3}$ \\ Xiangbing Mao $\mathbb{D}^{1,2,3}$ and Bing $Y u \mathbb{D}^{1,2,3}$ \\ ${ }^{1}$ Key Laboratory of Animal Disease-Resistant Nutrition, Sichuan Province, China \\ ${ }^{2}$ Key Laboratory of Animal Disease-Resistant Nutrition, Ministry of Education, China \\ ${ }^{3}$ Animal Nutrition Institute, Sichuan Agricultural University, Yaian, 625014, China \\ Correspondence should be addressed to Bing Yu; ybingtian@163.com
}

Received 27 February 2019; Revised 10 April 2019; Accepted 15 April 2019; Published 24 April 2019

Academic Editor: Gang Liu

Copyright (c) 2019 Yaolian Hu et al. This is an open access article distributed under the Creative Commons Attribution License, which permits unrestricted use, distribution, and reproduction in any medium, provided the original work is properly cited.

Dysbiosis and oxidative stress in the gut have contributed to the progression of intestinal inflammatory bowel disease (IBD). The current study has reported that enteric bacteria mediate redox homeostasis through the regulation of reactive oxygen species (ROS) production. Resveratrol, one of the most abundant polyphenols, with poor oral bioavailability, is considered as a scavenger of ROS and other free radicals. Recent studies have shown that resveratrol effectively enhances the growth of Lactococcus lactis and inhibits the growth of Enterococcus faecalis. (1) In terms of the two-way relationship between gut microbiota and resveratrol, resveratrol modulates gut microbiota; (2) in terms of resveratrol biotransformation by gut microbiota, we speculate that gut microbiota could be a target of resveratrol to maintain gut homeostasis. Here, we reviewed the current researches about the cellular signaling pathways in intestinal epithelial cells triggered by gut microbiota in response to oxidative stress. These results suggest that the modulation of the gut microbiota through resveratrol supplementation appears as a promising potential approach for the therapy of inflammatory bowel disease.

\section{Introduction}

Oxidative stress is caused by an imbalance between reactive oxygen species (ROS) production and cellular antioxidant capacity with accumulation of excessive ROS in cells $[1,2]$. Prolonged oxidative stress plays a key role in the initiation and development of inflammatory bowel disease (IBD), including Crohn's disease (CD) and ulcerative colitis (UC) [3-5]. IBD is an inappropriate immune response caused by a range of genetic, microbial, and environmental factors, characterized by chronic inflammation with alternating periods of remissions and relapses [6-8]. Recent evidence further identified that oxidative stress also appears to play a pathogenic role in chronic inflammatory diseases [9-11]. The inhibition of NF-E2 related factor-2 (Nrf2) attenuated anti-oxidative stress pathway and induced inflammation in Nrf2 knockout mice [12]. The intestinal mucosae of patients with IBD have high levels of ROS and decreased antioxidant defense capacity [13]. Excessive production of ROS can increase membrane permeability and cellular stress, finally resulting in the expansion of facultative anaerobic bacteria, gut barrier dysfunction, and inflammation [14, 15]. Moreover, experimental UC in mice is attenuated by antioxidant interventions [16]. Notably, certain intestinal epithelial cells have been reported to rapidly generate ROS in response to microbial signals [17]. The enteric bacteria in the intestinal lumen have been identified as a large source of redoxbased effector signals. They regulate multiple cell signaling via promoting the generation of ROS in intestinal epithelial cells, leading to the initiation and progression of many diseases such as IBD, metabolic syndrome, and cancer [18, 19]. The pathogenic bacteria-induced ROS will cause DNA damage in epithelial cells, which can give rise to genomic instability and the dysregulation of epithelial barrier function 
$[20,21]$. It is generally accepted that chronic inflammation is associated with the inability of the gut immune system to manage microflora and the alteration in the microbial composition in the gastrointestinal tract [22-25]. Furthermore, the metabolism disorder of bacterial fermentation products short-chain fatty acids (SCFAs) may stimulate the immune responses, resulting in increased intestinal epithelial permeability [26]. In all, the ROS overproduction in intestinal epithelial cells is the main consequence of intestinal dysbiosis that can be considered a significant risk factor of IBD. Thus, targeting gut microbiota to improve of oxidative stress and inflammation in the gut could be a promising potential therapy for IBD.

Resveratrol (3,5,4' -trihydroxy-trans-stilbene), a nonflavonoid polyphenol compound, is found in various plants, including Vitis vinifera, Arachis hypogaea, and Polygonum cuspidatum $[27,28]$. It is proved to be a potent antioxidant, antibacterial, antiobesity, anti-inflammatory, and anticancer agent in in vitro and in vivo experiments [29-33]. Notably, resveratrol is considered as a strong scavenger of ROS and other free radicals [34]. In a review, Manach et al. [35] proposed that polyphenols are the major dietary antioxidants present in the colon, as other antioxidants, such as vitamins $\mathrm{E}$ and $\mathrm{C}$, are absorbed in the small intestine. Although resveratrol has shown beneficial effects on host metabolism, measurements of plasma levels of the parent drug suggest that resveratrol exhibits poor bioavailability and high rate of metabolism when administered orally $[36,37]$. Resveratrol can be metabolized by hepatic and gut microbial enzymes $[38,39]$. The metabolite of resveratrol, dihydroresveratrol (DH-RES), is formed in cecum, colon, and rectum by gut microbiota fermentation, and the level of DH-RES is much higher than that of resveratrol. Therefore, this metabolite may also contribute to pharmacological activity in the human large intestine [36]. Besides, many studies have assessed the effect of resveratrol on gut microbiota diversity and composition, including inhibiting the growth of Enterococcus faecalis, increasing the Bacteroidetes-to-Firmicutes ratios, and increasing the Lactobacillus and Bifidobacterium populations $[40,41]$. Here, we discuss the cellular signaling triggered by enteric bacteria in host defense and disease development. In addition, we review the reciprocal interactions between resveratrol and gut microbiota, as well as the new evidence for the treatment of oxidative stress and inflammatory bowel disease.

\section{The Role of Gut Microbiota in IBD}

The intestine contains a complex microbial community of 100 trillion bacterial cells with more than 1,000 species, most of which are beneficial to our health $[42,43]$. The gut microbiota affect human metabolism due to their ability to interact with receptors on gut epithelial cells and other effector cells $[25,44,45]$. Given the development of novel diagnostic and therapeutic approaches for human diseases, a critical need exists for a deeper understanding of the underlying mechanism of cellular signaling induced by gut microbiota, and the symbiotic relationship between gut microbiota and the host.
In response to symbiotic microbes, intestinal epithelial cells can use NADPH oxidase (NOX) family to generate ROS, which have been detected in different multicellular organisms [46, 47]. NOX-produced $\mathrm{H}_{2} \mathrm{O}_{2}$, a major nonradical ROS production in the epithelial cells after the formylated peptide receptors sense and bind to a specific $\mathrm{N}$-formyl group of the Lactobacilli, is well documented as a second messenger in signal transduction networks. These changes in $\mathrm{H}_{2} \mathrm{O}_{2}$ do not cause a significant imbalance between oxidant production and antioxidant levels [48-50]. $\mathrm{H}_{2} \mathrm{O}_{2}$ generated by the cells can oxidize redox-sensitive cysteine residues on Kelch-like $\mathrm{ECH}$-associated protein 1 (Keap1), resulting in the activation of Nrf2 (see Figure 1) [51]. Of note, high levels of $\mathrm{H}_{2} \mathrm{O}_{2}$ further oxidize thiolate anions of the peroxidatic Cys to sulfinic $\left(\mathrm{SO}_{2} \mathrm{H}\right)$ or sulfonic $\left(\mathrm{SO}_{3} \mathrm{H}\right)$ species. This irreversible modification can lead to cellular damage and oxidative stress [52]. In fact, the diverse biological outcomes of different ROS depend on the specificity and selectivity of ROS on their targets and the compartmentalization of ROS production in cells [53]. Lactococcus lactis was found to diminish oxidative stress, by releasing cytoplasmic superoxide dismutase A (SodA) due to host lysozyme-mediated lysis at inflamed colonic sites [54]. Interestingly, Lactobacillus appears to modify the natural course of the disease through the upregulation of Nrf2dependent antioxidant enzymes. Enterococcus faecalis near the oxygenated colonic luminal surface generates extracellular $\mathrm{O}^{2-}$ at high rate causing intestinal injury $[23,55]$. For example, germ-free interleukin-10 knockout (IL-10 KO) mice developed IBD after colonization with Enterococcus faecalis but not in the Lactococcus lactis colonized mice [56]. Indeed, unlike Lactococcus lactis, intestinal ROS overproduction is induced by opportunistic pathogens under certain conditions, and this can aggravate intestinal damage. The bacterialderived uracil is responsible for dual oxidase (DUOX)dependent ROS generation in human mucosal epithelial cells, which can lead to overexpression of DUOX2 and oxidative stress in the gut (see Figure 1) [57]. Oxygen radical generation by opportunistic pathogens promotes epithelial cell DNA damage and exacerbates intestine inflammation [58]. Taken together, the microbiota composition is thought to be a major determinant of health and disease of the host, because of the potential of intestinal microbiota to modulate ROS production and antioxidant defense.

Culture-independent methods to analyze the gut microbial composition allow a more detailed understanding of the alterations in gut microbe and IBD. The high levels of mucosa-associated bacteria were detected in those patients [59, 60]. As mentioned above, the studies have demonstrated an association between microbiota composition and redox state of enterocytes; that is, several enteric bacteria could produce extracellular ROS, which may dysregulate intestinal homeostasis (see Figure 1). Moreover, anaerobic intestinal bacteria can downregulate proinflammatory cytokines expression and modulate the host mucosal immune response by inhibiting peroxisome proliferatoractivated receptor gamma-mediated nuclear factor-kappaB $(\mathrm{NF}-\kappa \mathrm{B})$ activation $[61,62]$. Furthermore, anaerobic bacterial fermentation gives rise to the production of SCFAs, including acetate, propionate, and butyrate. SCFAs have been 


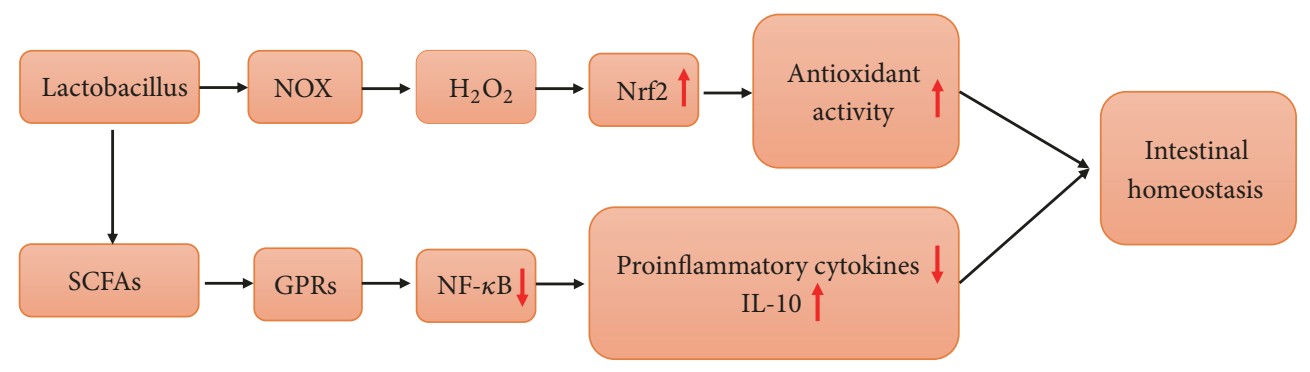

(a)

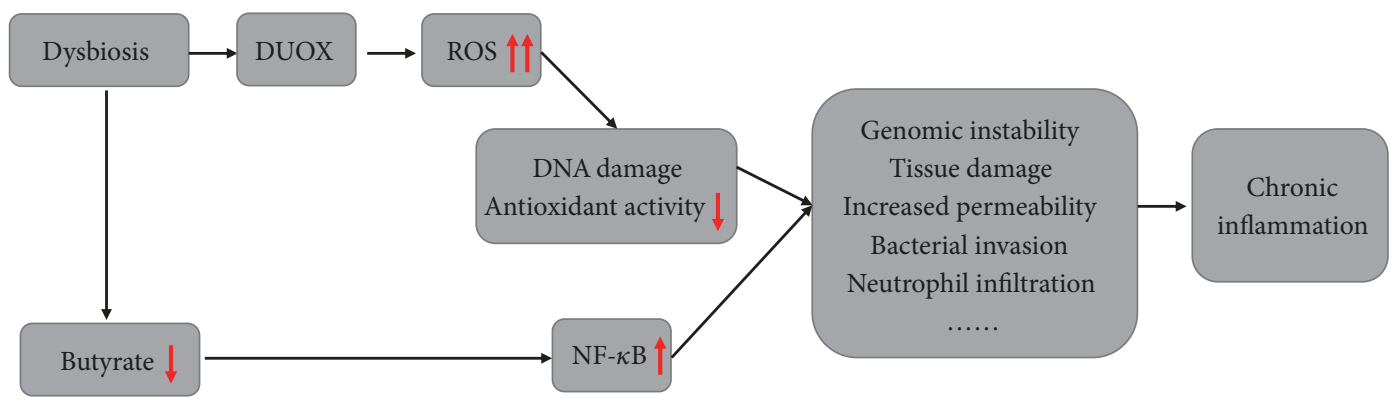

(b)

Figure 1: The associations between gut microbiota, intestinal inflammation, and oxidative stress. (a) Intestinal homeostasis is associated with enteric bacteria residing in the intestinal lumen. (b) Dysbiosis and oxidative stress in the gut have been shown as contributors of the pathogenesis of intestinal diseases.

suggested to bind to the intestinal epithelial cell-surface Gprotein-coupled receptors (GPRs) in the surface of intestinal epithelial cells, such as GPR41, GPR43, and GPR109A [6366]. They maintain intestinal homeostasis by suppressing NF$\kappa \mathrm{B}$ activation, reducing the production of proinflammatory factors, increasing intestinal mucus synthesis, and decreasing the intestinal epithelial permeability (see Figure 1) [67-69]. Moreover, the metabolism of butyrate in the colonocytes has been shown to drive the oxygen consumption, which leads to a significant reduction of $\mathrm{H}_{2} \mathrm{O}_{2}$-induced DNA damage $[26,70]$. A hypoxic environment induced by the oxygen consumption is also essential for preventing the expansion of facultative anaerobic bacteria. However, continued oxidative stress can alter the colonocyte metabolism and damage the hypoxic environment in large intestine, leading to the expansion of facultative anaerobic bacteria during dysbiosis [71]. The disorder of butyrate metabolism can give rise to an increasing number of macrophages and NF- $\kappa \mathrm{B}$ activation in colonocytes of patients with UC (see Figure 1) [72].

Several clinical studies have shown that the fecal microbiota transplantation (FMT) from the healthy donor promotes intestinal microbiota recovery and inflammation resolution in IBD patients $[73,74]$. Therefore, a new potent approach by normalizing the intestinal dysbiosis and improving redox imbalance may open a new era for patients with chronic inflammation in the gut.

\section{The Relationship between Resveratrol and Gut Microbiota}

Resveratrol and its derivatives have shown therapeutic potential for the prevention and treatment of different chronic diseases such as diabetes and IBD [75-78]. Resveratrol, as a phytoalexin, shows antioxidant and anti-inflammatory activities to improve oxidative stress and chronic inflammation $[30,79]$. For instance, resveratrol protects the porcine intestinal epithelial cell line (IPEC-J2) from mycotoxininduced increases in intracellular ROS level and cell damage through the regulation of the Nrf2 signaling pathway [80]. The supplementation with $500 \mathrm{mg}$ resveratrol can reduce levels of proinflammatory mediators and inhibit activity of $\mathrm{NF}-\kappa \mathrm{B}$ in patients with active UC [81]. However, the low water solubility of resveratrol leads to poor oral bioavailability, which has limits for resveratrol's concentration in plasma $[82,83]$. It seems to be a controversial issue about multiple biological activities of resveratrol. It can be speculated that enteric bacteria might represent a major contributor to the effects of resveratrol on the functional modifications of host cells $[35,84]$. Using ultrahigh-performance liquid chromatography (UHPLC), coupled with a linear ion trap mass spectrometer, this hypothesis has been confirmed by these determinations of resveratrol metabolites in plasma and colon. Resveratrol was shown to be metabolized into various derivatives by pharmacokinetic profiling [85]. Resveratrol cannot be absorbed in the native form but is present at a very low concentration in plasma in conjugated forms such as sulfate and glucuronide conjugates [86-88]. An accumulation of resveratrol in the large intestine after oral administration has been reported, because of its poor absorption [37, 39]. It has been reported that resveratrol can modulate gut microbial composition while microbiota can also regulate resveratrol biotransformation $[89,90]$.

However, it is debated whether the effects of resveratrol on gut microbiome modulation are enough to be responsible 
TABLE 1: The effects of resveratrol administration are partly dependent on the gut microbiota.

\begin{tabular}{|c|c|c|c|c|}
\hline Animal model & Treatment & Alerted bacterial taxa & Biological effects & Reference \\
\hline Kunming mice & High-fat diet & $\begin{array}{l}\text { Increasing Lactobacillus and } \\
\text { Bifidobacterium abundance; } \\
\text { decreasing Enterococcus faecalis } \\
\text { abundance }\end{array}$ & Anti-obesity effects & $\begin{array}{l}\text { Qiao et al. } \\
\quad[41]\end{array}$ \\
\hline Wistar rats & $\begin{array}{c}\text { High-fat sucrose } \\
\text { diet }\end{array}$ & $\begin{array}{l}\text { Decreasing the abundance of } \\
\text { Parabacteroides genus }\end{array}$ & $\begin{array}{l}\text { Altering the mRNA expression of } \\
\text { tight-junction proteins and } \\
\text { inflammation-associated genes }\end{array}$ & $\begin{array}{c}\text { Etxeberria et } \\
\text { al. }[90]\end{array}$ \\
\hline Fischer F344 rats & DSS & $\begin{array}{l}\text { Increasing Lactobacillus and } \\
\text { Bifidobacterium abundance; } \\
\text { decreasing Enterococcus faecalis } \\
\text { abundance }\end{array}$ & $\begin{array}{l}\text { Protecting the colonic mucosa } \\
\text { architecture and reducing } \\
\text { systemic inflammation markers }\end{array}$ & $\begin{array}{l}\text { Larrosa et al. } \\
\text { [84] }\end{array}$ \\
\hline Wistar rats & High-fat diet & $\begin{array}{l}\text { Inhibiting the growth of } \\
\text { Bacteroides and } \\
\text { Desulfovibrionaceae sp.; } \\
\text { enhancing the proportion of } \\
\text { Blautia and Dorea in the } \\
\text { Lachnospiraceae family }\end{array}$ & $\begin{array}{c}\text { Reducing fasting blood glucose } \\
\text { levels and increasing the HDL-c } \\
\text { levels }\end{array}$ & $\begin{array}{l}\text { Yang et al. } \\
\quad[91]\end{array}$ \\
\hline C57BL/6J mice & $\begin{array}{l}\text { Choline or } \\
\text { Trimethylamine }\end{array}$ & $\begin{array}{c}\text { Increasing the relative abundance } \\
\text { of Bacteroides, Lactobacillus, } \\
\text { Bifidobacterium, and } \\
\text { Akkermansia; decreasing the } \\
\text { relative abundance of Prevotella, } \\
\text { uncultured Ruminococcaceae, } \\
\text { Anaerotruncus, Alistipes, } \\
\text { Helicobacter, and uncultured } \\
\text { Peptococcaceae }\end{array}$ & Anti-atherosclerosis effects & $\begin{array}{l}\text { Chen et al. } \\
\text { [93] }\end{array}$ \\
\hline
\end{tabular}

for its antioxidant and anti-inflammatory activities and these effects are associated with the improvement of gut diseases in the process of bacteria-mediated ROS overproduction. The definitive mechanisms remain to be obtained. Based on the current evidence, the potential mechanisms will be discussed in the following sections.

\subsection{Alterations of the Intestinal Microbiota by Resveratrol.} Resveratrol is known to modulate the composition of the gut microbiota with a decrease in opportunistic pathogenic bacteria in vivo. Yang et al. [91] showed a significant decrease in the diversity of the gut microbiota and an increase in oxidative stress in high-fat-diet-fed rats compared with that in controls. Resveratrol supplementation (400 mg resveratrol per $\mathrm{kg}$ of feed for 8 weeks) increases the population of butyrate producer Blautia and Dorea in the Lachnospiraceae family. However, they noted that the supplementation of resveratrol had no significant influence on the levels of SCFAs. SCFAs are rapidly absorbed in the colon. This may result in the inaccuracy of SCFAs measurement. Additionally, resveratrol administration decreased the population of Bacteroidetes. Most Bacteroides, as antibiotic-resistant bacteria, may become highly pathogenic bacteria. The increased levels of Bacteroides can result in inflammation [92]. However, Qiao et al. [41] demonstrated that resveratrol supplementation (200 $\mathrm{mg} / \mathrm{kg} / \mathrm{d}$ for 12 weeks) promotes a higher Bacteroidetes-toFirmicutes ratio in Western-diet-fed mice. Species (rats vs. mice) and/or housing environments (isolated vs. conventional) being studied could potentially explain the differences in specific microbial changes. In addition, the number of Lactobacillus and Bifidobacterium was significantly increased in resveratrol-fed animals. These genera, as noted above, are closely linked with redox signaling in mucosal epithelial cells, which plays a critical role in maintaining gut homeostasis. Moreover, Enterococcus faecalis, which is linked with the high levels of extracellular $\mathrm{O}^{2-}$, was significantly decreased in resveratrol-fed mice. Similarly, recent evidence suggests that the relative abundance of Bacteroides, Lactobacillus, Bifidobacterium, and Akkermansia is increased with resveratrol supplementation [93]. Previous studies have also shown that resveratrol administration leads to the increases in the number of Lactobacilli and the decrease in the number of Enterococcus faecalis and Escherichia coli species in high-fatfed mice. Both Escherichia coli and Enterococcus faecalis are positively correlated with colonic ROS and MDA levels, while several bacteria such as Lactobacilli are significantly associated with colonic T-AOC [94]. Cellular studies also indicated an antimicrobial activity of natural phenolic compounds such as resveratrol and kaempferol, which is a growth inhibition of Enterococcus faecalis [95]. Thus, it is speculated that resveratrol alleviates oxidative stress by ROS and subsequent intestinal damage through gut microbiota (see Table 1).

A previous study, using the dextran sulfate sodiuminduced colitis (DSS-colitis) rat model, has demonstrated that resveratrol treatment $(1 \mathrm{mg} / \mathrm{kg} /$ day for 25 days $)$ provides beneficial effects on the colon, including altering the expression of inflammation-associated genes, protecting the colonic mucosa architecture, and modulating intracellular 
signaling such as NF- $\kappa \mathrm{B}$ signaling pathway [84]. In addition, resveratrol administration can also restore normal intestinal microbiota in bacterial composition under DSS treatment. These include some anti-inflammatory gut microbiota, such as Lactobacilli and Bifidobacteria. More recently, Wellman et al. [96] reported that aged mice with deletion of Sirtuin1 in the intestinal epithelium (SIRT1 iKO mice) exhibited a reduced abundance of Bacilli, particularly Lactobacillus. Compared to wild-type DSS-colitis mice, aged SIRT1 iKO mice experienced enhanced rectal bleeding, increased colonic shortening, and elevated colonic crypt erosion after DSS challenge. Therefore, SIRT1 has been shown to improve intestinal barrier function and maintain epithelial cell homeostasis. Interestedly, depletion of gut microbiota by the antibiotic cocktail in SIRT1 iKO mice shows minimal colonic spontaneous inflammation. Hence, SIRT1 deficiency-induced intestinal mucosal damage is highly associated with alterations in gut microbiota upon DSS treatment. In view of the proved associations between gut microbiota, intestinal inflammation, and SIRT1, among them, gut microbiota is the main target of SIRT1 to reduce inflammation markers. Resveratrol, a well-known activator of SIRT1, has been shown to significantly increase SIRT1 activity $[97,98]$. It has been postulated that health benefits of resveratrol in the gut are largely dependent on the gut microbiota.

\subsection{Antioxidant and Anti-Inflammatory Properties of Resvera-} trol-Derived Microbial Metabolites. As mentioned previously, large quantities of resveratrol and its metabolites were found in the content of gastrointestinal tract in resveratrol-fed animals. The new data suggested that most unabsorbed resveratrol was transformed by gut microbiota into various bioactive metabolites, including DH-RES, 3,4'-dihydroxytrans-stilbene, and piceid [99]. The enzymes of these bacteria, such as Slackia equolifaciens and Adlercreutzia equolifaciens, are responsible for the hydrogenation of resveratrol to yield DH-RES [100]. In addition, Jung et al. [101] showed that DH-RES was also synthesized by E. lenta ATCC 43055 and B. uniformis ATCC 8492 in vitro. For piceid, studies have demonstrated that Bacillus cereus could transform resveratrol into piceid. Of note, though the biological activity of resveratrol is generally attributed to the parent drug, bacterial-derived metabolites have demonstrated equal or similar activity.

In in vitro experiments, Lin et al. [102] indicate that 3,4'dihydroxy-trans-stilbene has cytoprotection function against t-BHP-induced oxidative insult through activating the Keap1Nrf2-ARE signaling pathway and the downstream antioxidant genes in HepG2 cells. This bacterial metabolite exhibits similar antioxidant effects in gut tract. Moreover, recent evidence has established that DH-RES, whose solubility is higher than that of resveratrol, can participate in the inhibition of NF- $\kappa \mathrm{B}$ activity in the cerulein-treated rats [103]. Piceid and resveratrol show similar activities as inhibitors of the lipid peroxidation. Piceid may be more efficacious than resveratrol due to the slow reactivity [104]. Piceid administration can alter the transfer of electrons from NADPH to oxygen and the production of ROS, increasing the availability of GSH and the activity of SOD in rotenone-induced Parkinson's disease rat models [105]. Plant callus is considered as a source of valuable secondary metabolites. Several studies have reported that rice callus suspension culture (RCSC) exhibits strong antiinflammatory and antiproliferative activity. RCSC treatment has been shown to exhibit strong ROS modulating effects and reduce the effect of inflammation on cell death in cell lines treated with the proinflammatory cytokine cocktail. Piceid, 4-deoxyphloridzin, $5^{\prime}$-methoxycurcumin and lupeol, identified through HPLC and mass spectroscopy, are responsible for biological activities of RCSC [106]. Zhu et al. [107] demonstrated that DH-RES, an extract of Dendrobium, quenches intracellular ROS in a more efficient manner than vitamin E. In experimental acute pancreatitis rats, the levels of proinflammatory cytokines were notably reduced, and the nuclear expression of NF- $\kappa \mathrm{B}$ was remarkably decreased with the administration of DH-RES at 10,20 , and $50 \mathrm{mg} / \mathrm{kg}$ [108]. Furthermore, Kim et al. [109] recently reported that resveratrol-FMT recipients, which are obese mice receiving FMTs from healthy resveratrol-fed mice, showed significantly lower inflammatory cytokine levels in the colon when compared with Chow-FMT recipients. These results indicated that resveratrol and/or its metabolites suppressed mucosal inflammation through the inhibition of NF- $\kappa \mathrm{B}$ activation. Sung et al. [110] previously demonstrated that bacterialderived metabolites induced by resveratrol are capable of modulating energy metabolism in high-fat high-salt-fed mice receiving FMTs and increasing SCFAs production. It is suggested that the biological activity of metabolites or nonliving microorganisms in the gut is also associated with SCFAs production.

\section{Conclusions}

Gut microbiota regulates the cellular redox state in the host organism. Lactobacillus-mediated ROS production within gut epithelial cells in low levels maintains gut homeostasis, whereas high levels of extracellular O2- induced by Enterococcus faecalis cause epithelial cell DNA damage, intestinal injury, and inflammatory responses. Additionally, proinflammatory cytokines expression and the production of SCFAs, which are associated with enteric bacteria, can both modulate the proinflammatory NF- $\kappa$ B signaling pathway.

Resveratrol can inhibit inflammatory disorders through the changes in the gut microbiota. Resveratrol and its microbial metabolites can reduce the increased levels of ROS, activate Nrf2 signaling, and improve oxidative stress. They protect epithelial barrier function and suppress the activation of $\mathrm{NF}-\kappa \mathrm{B}$ and intestinal inflammation. However, questions remain regarding the bidirectional interactions between resveratrol and gut microbiota, and if these interactions can fully afford resveratrol's biological activity. The mechanism of how resveratrol and its derivatives regulate ROS production in intestinal epithelial cells has yet to be fully elucidated. Considering the health benefits of resveratrol and its metabolic characteristics, the administration of resveratrol is a novel and rational strategy for the treatment of chronic inflammatory diseases. 


\section{Conflicts of Interest}

There are no conflicts of interest regarding this manuscript.

\section{Acknowledgments}

This work was supported by the Key Program of the National Natural Science Foundation of China (31730091). We thank Dr. Hui Yan of Washington University in St. Louis for editing the manuscript and are grateful to Yong Zhang and Jin Wan of Sichuan Agricultural University for helpful advice.

\section{References}

[1] A. Abdal Dayem, H.-Y. Choi, J.-H. Kim, and S.-G. Cho, "Role of oxidative stress in stem, cancer, and cancer stem cells," Cancers, vol. 2, no. 2, pp. 859-884, 2010.

[2] P. Newsholme, V. F. Cruzat, K. N. Keane, R. Carlessi, and P. I. de Bittencourt, "Molecular mechanisms of ROS production andoxidative stress in diabetes," Biochemical Journal, vol. 473, no. 24, pp. 4527-4550, 2016.

[3] J. Li, H. Chen, B. Wang et al., " $\mathrm{ZnO}$ nanoparticles act as supportive therapy in DSS-induced ulcerative colitis in mice by maintaining gut homeostasis and activating Nrf2 signaling," Scientific Reports, vol. 7, Article ID 43126, 2017.

[4] E. Dudzińska, M. Gryzinska, K. Ognik, P. Gil-Kulik, and J. Kocki, "Oxidative stress and effect of treatment on the oxidation product decomposition processes in IBD," Oxidative Medicine and Cellular Longevity, vol. 2018, no. 4, pp. 1-7, 2018.

[5] R. D’Incà, R. Cardin, L. Benazzato, I. Angriman, D. Martines, and G. C. Sturniolo, "Oxidative DNA damage in the mucosa of ulcerative colitis increases with disease duration and dysplasia," Inflammatory Bowel Diseases, vol. 10, no. 1, pp. 23-27, 2004.

[6] F. Zhang, Y. Li, X. Wang, S. Wang, and D. Bi, "The impact of lactobacillus plantarum on the gut microbiota of mice with DSS-induced colitis," BioMed Research International, vol. 2019, Article ID 3921315, 10 pages, 2019.

[7] Z. Hong and M. Piao, "Effect of quercetin monoglycosides on oxidative stress and gut microbiota diversity in mice with dextran sodium sulphate-induced colitis," BioMed Research International, vol. 2018, Article ID 8343052, 7 pages, 2018.

[8] L. Huang, R. Gao, N. Yu, Y. Zhu, Y. Ding, and H. Qin, "Dysbiosis of gut microbiota was closely associated with psoriasis," SCIENCE CHINA Life Sciences, 2018.

[9] Guiping Guan and Shile Lan, "Implications of antioxidant systems in inflammatory bowel disease," BioMed Research International, vol. 2018, Article ID 1290179, 7 pages, 2018.

[10] J. Chen, B. Yu, D. Chen et al., "Chlorogenic acid improves intestinal barrier functions by suppressing mucosa inflammation and improving antioxidant capacity in weaned pigs," The Journal of Nutritional Biochemistry, vol. 59, pp. 84-92, 2018.

[11] L. Kruidenier and H. W. Verspaget, "Oxidative stress as a pathogenic factor in inflammatory bowel disease-radicals or ridiculous?" Alimentary Pharmacology \& Therapeutics, vol. 16, no. 12, pp. 1997-2015, 2002.

[12] K. L. Cheung, J. H. Lee, T. O. Khor et al., "Nrf2 knockout enhances intestinal tumorigenesis in Apcmin/+ mice due to attenuation of anti-oxidative stress pathway while potentiates inflammation," Molecular Carcinogenesis, vol. 53, no. 1, pp. 7784, 2014.
[13] D. S. Wilson, G. Dalmasso, L. Wang, S. V. Sitaraman, D. Merlin, and N. Murthy, "193 orally delivered thioketal-nanoparticles loaded with TNF $\alpha$-SiRNA target inflammation and inhibit gene expression in the intestines," Gastroenterology, vol. 138, no. 5, pp. S-35-S-36, 2010.

[14] M.-K. Sung, J.-Y. Yeon, S.-Y. Park, J. H. Y. Park, and M.-S. Choi, "Obesity-induced metabolic stresses in breast and colon cancer," Annals of the New York Academy of Sciences, vol. 1229, no. 1, pp. 61-68, 2011.

[15] X. Mao, Q. Yang, D. Chen, B. Yu, and J. He, "Benzoic acid used as food and feed additives can regulate gut functions," BioMed Research International, vol. 2019, Article ID 5721585, 6 pages, 2019.

[16] A. E. Khodir, E. Said, H. Atif, H. A. ElKashef, and H. A. Salem, "Targeting Nrf2/HO-1 signaling by crocin: Role in attenuation of AA-induced ulcerative colitis in rats," Biomedicine \& Pharmacotherapy, vol. 110, pp. 389-399, 2019.

[17] A. Kumar, H. Wu, L. S. Collier-Hyams et al., "Commensal bacteria modulate cullin-dependent signaling via generation of reactive oxygen species," EMBO Journal, vol. 26, no. 21, pp. 4457-4466, 2007.

[18] W.-J. Lee, "Bacterial-modulated signaling pathways in gut homeostasis," Science Signaling, vol. 1, no. 21, article pe24, 2008.

[19] P. J. Daschner, M. B. Grisham, and M. G. Espey, "Redox relationships in gut-microbiome interactions," Free Radical Biology \& Medicine, vol. 105, pp. 1-2, 2017.

[20] J.-P. Nougayrède, S. Homburg, F. Taieb et al., "Escherichia coli induces DNA double-strand breaks in eukaryotic cells," Science, vol. 313, no. 5788, pp. 848-851, 2006.

[21] Y. Liu, H. J. Van Kruiningen, A. B. West, R. W. Cartun, A. Cortot, and J.-F. Colombel, "Immunocytochemical evidence of Listeria, Escherichia coil, and Streptococcus antigens in Crohn's disease," Gastroenterology, vol. 108, no. 5, pp. 1396-1404, 1995.

[22] I. Koboziev, W. C. Reinoso, K. L. Furr, and M. B. Grisham, "Role of the enteric microbiota in intestinal homeostasis and inflammation," Free Radical Biology \& Medicine, vol. 68, pp. 122-133, 2014.

[23] M. M. Huycke, V. Abrams, and D. R. Moore, "Enterococcus faecalis produces extracellular superoxide and hydrogen peroxide that damages colonic epithelial cell DNA," Carcinogenesis, vol. 23, no. 3, pp. 529-536, 2002.

[24] P. Bercik, E. Denou, J. Collins et al., "The intestinal microbiota affect central levels of brain-derived neurotropic factor and behavior in mice," Gastroenterology, vol. 141, no. 2, article e593, pp. 599-609, 2011.

[25] W. Ren, P. Wang, J. Yan et al., "Melatonin alleviates weanling stress in mice: Involvement of intestinal microbiota," Journal of Pineal Research, vol. 64, no. 2, Article ID e12448, 2018.

[26] H. M. Hamer, D. M. A. E. Jonkers, A. Bast et al., "Butyrate modulates oxidative stress in the colonic mucosa of healthy humans," Clinical Nutrition, vol. 28, no. 1, pp. 88-93, 2009.

[27] L. Frémont, "Biological effects of resveratrol," Life Sciences, vol. 66, no. 8, pp. 663-673, 2000.

[28] J. A. Baur and D. A. Sinclair, "Therapeutic potential of resveratrol: the in vivo evidence," Nature Reviews Drug Discovery, vol. 5, no. 6, pp. 493-506, 2006.

[29] A. Malhotra, S. Bath, and F. Elbarbry, "An organ system approach to explore the antioxidative, anti-inflammatory, and cytoprotective actions of resveratrol," Oxidative Medicine and Cellular Longevity, vol. 2015, Article ID 803971, 15 pages, 2015. 
[30] C. A. de La Lastra and I. Villegas, "Resveratrol as an antioxidant and pro-oxidant agent: mechanisms and clinical implications," Biochemical Society Transactions, vol. 35, no. 5, pp. 1156-1160, 2007.

[31] M. M.-Y. Chan, "Antimicrobial effect of resveratrol on dermatophytes and bacterial pathogens of the skin," Biochemical Pharmacology, vol. 63, no. 2, pp. 99-104, 2002.

[32] S. Y. Kim, Y. J. Jin, Y. S. Choi, and T. Park, "Resveratrol exerts anti-obesity effects via mechanisms involving down-regulation of adipogenic and inflammatory processes in mice," Biochemical Pharmacology, vol. 81, no. 11, pp. 1343-1351, 2011.

[33] L. Huminiecki and J. Horbańczuk, "The functional genomic studies of resveratrol in respect to its anti-cancer effects," Biotechnology Advances, vol. 36, no. 6, pp. 1699-1708, 2018.

[34] S. S. Leonard, C. Xia, B.-H. Jiang et al., "Resveratrol scavenges reactive oxygen species and effects radical-induced cellular responses," Biochemical and Biophysical Research Communications, vol. 309, no. 4, pp. 1017-1026, 2003.

[35] C. Manach, A. Scalbert, C. Morand, C. Rémésy, and L. Jiménez, "Polyphenols: food sources and bioavailability," American Journal of Clinical Nutrition, vol. 79, no. 5, pp. 727-747, 2004.

[36] T. Walle, F. Hsieh, M. H. DeLegge, J. E. Oatis Jr., and U. K. Walle, "High absorption but very low bioavailability of oral resveratrol in humans," Drug Metabolism and Disposition, vol. 32, no. 12, pp. 1377-1382, 2004.

[37] M. Azorín-Ortuño, M. J. Yañéz-Gascón, and F. J. Pallarés, "Pharmacokinetic study of trans-resveratrol in adult pigs," Journal of Agricultural and Food Chemistry, vol. 58, no. 20, pp. 11165-11171, 2010.

[38] A. Amri, J. C. Chaumeil, S. Sfar, and C. Charrueau, "Administration of resveratrol: what formulation solutions to bioavailability limitations?" Journal of Controlled Release, vol. 158, no. 2, pp. 182-193, 2012.

[39] X. Vitrac, A. Desmoulière, B. Brouillaud et al., "Distribution of [14C]-trans-resveratrol, a cancer chemopreventive polyphenol, in mouse tissues after oral administration," Life Sciences, vol. 72, no. 20, pp. 2219-2233, 2003.

[40] L. Zhao, Q. Zhang, W. Ma, F. Tian, H. Shen, and M. Zhou, "A combination of quercetin and resveratrol reduces obesity in high-fat diet-fed rats by modulation of gut microbiota," Food \& Function, vol. 8, no. 12, pp. 4644-4656, 2017.

[41] Y. Qiao, J. Sun, S. Xia, X. Tang, Y. Shi, and G. Le, "Effects of resveratrol on gut microbiota and fat storage in a mouse model with high-fat-induced obesity," Food \& Function, vol. 5, no. 6, pp. 1241-1249, 2014.

[42] J. C. Clemente, L. K. Ursell, L. W. Parfrey, and R. Knight, “The impact of the gut microbiota on human health: an integrative view," Cell, vol. 148, no. 6, pp. 1258-1270, 2012.

[43] K. Ikuma, A. W. Decho, and B. Lau, "The extracellular bastions of bacteria ${ }^{\mathrm{TM}}$ a biofilm way of life," Nature Education Knowledge, vol. 4, no. 2, pp. 2-19, 2013.

[44] S. A. Joyce and C. G. M. Gahan, "The gut microbiota and the metabolic health of the host," Current Opinion in Gastroenterology, vol. 30, no. 2, pp. 120-127, 2014.

[45] L. Weichselbaum and O. D. Klein, "The intestinal epithelial response to damage," Science China Life Sciences, pp. 1-7, 2018.

[46] R. M. Jones, L. Luo, C. S. Ardita et al., "Symbiotic lactobacilli stimulate gut epithelial proliferation via Nox-mediated generation of reactive oxygen species," EMBO Journal, vol. 32, no. 23, pp. 3017-3028, 2013.
[47] J. D. Lambeth, "NOX enzymes and the biology of reactive oxygen," Nature Reviews Immunology, vol. 4, no. 3, pp. 181-189, 2004.

[48] S. Voltan, D. Martines, M. Elli et al., "Lactobacillus crispatus M247-derived $\mathrm{H} 2 \mathrm{O} 2$ acts as a signal transducing molecule activating peroxisome proliferator activated receptor-gamma in the intestinal mucosa," Gastroenterology, vol. 135, no. 4, pp. 1216-1227, 2008.

[49] I. Saxena, S. Srikanth, and Z. Chen, "Cross talk between $\mathrm{H} 2 \mathrm{O} 2$ and interacting signal molecules under plant stress response," Frontiers in Plant Science, vol. 7, no. 22, 2016.

[50] A. S. Neish, "Redox signaling mediated by the gut microbiota," Free Radical Research, vol. 47, no. 11, pp. 950-957, 2013.

[51] H. Bryan, Molecular Investigation of Keap1-Dependent Regulation of the Nrf2 Cell Defence Pathwa, University of Liverpool, 2014.

[52] M. Zaffagnini, M. Bedhomme, C. H. Marchand, S. Morisse, P. Trost, and S. D. Lemaire, "Redox regulation in photosynthetic organisms: focus on glutathionylation," Antioxidants \& Redox Signaling, vol. 16, no. 6, pp. 567-586, 2012.

[53] M. Schieber and N. S. Chandel, "ROS function in redox signaling and oxidative stress," Current Biology, vol. 24, no. 10, pp. R453-R462, 2014.

[54] S. A. Ballal, P. Veiga, K. Fenn et al., "Host lysozyme-mediated lysis of Lactococcus lactis facilitates delivery of colitisattenuating superoxide dismutase to inflamed colons," Proceedings of the National Acadamy of Sciences of the United States of America, vol. 112, no. 25, pp. 7803-7808, 2015.

[55] C. Reboul, J. Thireau, G. Meyer et al., "Free radical biology and medicine," Free Radical Biology \& Medicine, vol. 35, no. 5, 2003.

[56] E. Balish and T. Warner, "Enterococcus faecalis induces inflammatory bowel disease in interleukin-10 knockout mice," The American Journal of Pathology, vol. 160, no. 6, pp. 2253-2257, 2002.

[57] K.-A. Lee, S.-H. Kim, E.-K. Kim et al., "Bacterial-derived uracil as a modulator of mucosal immunity and gut-microbe homeostasis in drosophila," Cell, vol. 153, no. 4, pp. 797-811, 2013.

[58] M. Aslan, Y. Nazligul, C. Bolukbas et al., "Peripheral lymphocyte DNA damage and oxidative stress in patients with ulcerative colitis," Polskie Archiwum Medycyny Wewnętrznej, vol. 121, no. 7-8, pp. 223-229, 2011.

[59] C. D. Packey and R. B. Sartor, "Commensal bacteria, traditional and opportunistic pathogens, dysbiosis and bacterial killing in inflammatory bowel diseases," Current Opinion in Infectious Diseases, vol. 22, no. 3, pp. 292-301, 2009.

[60] C. W. Png, S. K. Lindén, K. S. Gilshenan et al., "Mucolytic bacteria with increased prevalence in IBD mucosa augment in vitro utilization of mucin by other bacteria," American Journal of Gastroenterology, vol. 105, no. 11, pp. 2420-2428, 2010.

[61] V. Tremaroli and F. Bäckhed, "Functional interactions between the gut microbiota and host metabolism," Nature, vol. 489, no. 7415, pp. 242-249, 2012.

[62] D. Kelly, J. I. Campbell, T. P. King et al., "Commensal anaerobic gut bacteria attenuate inflammation by regulating nuclearcytoplasmic shuttling of PPAR- $\gamma$ and RelA," Nature Immunology, vol. 5, no. 1, pp. 104-112, 2004.

[63] M. A. R. Vinolo, G. J. Ferguson, S. Kulkarni et al., "SCFAs induce mouse neutrophil chemotaxis through the GPR43 receptor," PLoS ONE, vol. 6, no. 6, 2011. 
[64] H. Tazoe, Y. Otomo, I. Kaji, R. Tanaka, S.-I. Karaki, and A. Kuwahara, "Roles of short-chain fatty acids receptors, GPR41 and GPR43 on colonic functions," Journal of Physiology and Pharmacology, vol. 59, no. 2, pp. 251-262, 2008.

[65] M. H. Kim, S. G. Kang, J. H. Park, M. Yanagisawa, and C. H. Kim, "Short-chain fatty acids activate GPR41 and GPR43 on intestinal epithelial cells to promote inflammatory responses in mice," Gastroenterology, vol. 145, no. 2, pp. 396.e10-406.e10, 2013.

[66] W.-J. Lee and K. Hase, "Gut microbiota-generated metabolites in animal health and disease," Nature Chemical Biology, vol. 10, no. 6, pp. 416-424, 2014.

[67] R. Masui, M. Sasaki, Y. Funaki et al., "G protein-coupled receptor 43 moderates gut inflammation through cytokine regulation from mononuclear cells," Inflammatory Bowel Diseases, vol. 19, no. 13, pp. 2848-2856, 2013.

[68] T. Hudcovic, R. Štěpánková, J. Cebra, and H. TlaskalováHogenová, "The role of microflora in the development of intestinal inflammation: acute and chronic colitis induced by dextran sulfate in germ-free and conventionally reared immunocompetent and immunodeficient mice," Folia Microbiologica, vol. 46, no. 6, pp. 565-572, 2001.

[69] N. Huda-Faujan, A. S. Abdulamir, A. B. Fatimah et al., "The impact of the level of the intestinal short chain Fatty acids in inflammatory bowel disease patients versus healthy subjects," The Open Biochemistry Journal, vol. 4, pp. 53-58, 2010.

[70] J. Sauer, K. K. Richter, and B. L. Pool-Zobel, "Physiological concentrations of butyrate favorably modulate genes of oxidative and metabolic stress in primary human colon cells," The Journal of Nutritional Biochemistry, vol. 18, no. 11, pp. 736-745, 2007.

[71] Y. Litvak, M. X. Byndloss, and A. J. Bäumler, "Colonocyte metabolism shapes the gut microbiota," Science, vol. 362, no. 6418, Article ID eaat9076, 2018.

[72] H. Lührs, T. Gerke, J. G. Müller et al., "Butyrate inhibits NF$\kappa \mathrm{B}$ activation in lamina propria macrophages of patients with ulcerative colitis," Scandinavian Journal of Gastroenterology, vol. 37, no. 4, pp. 458-466, 2002.

[73] J. L. Anderson, R. J. Edney, and K. Whelan, "Systematic review: faecal microbiota transplantation in the management of inflammatory bowel disease," Alimentary Pharmacology \& Therapeutics, vol. 36, no. 6, pp. 503-516, 2012.

[74] C. Staley, A. Khoruts, and M. J. Sadowsky, "Contemporary applications of fecal microbiota transplantation to treat intestinal diseases in humans," Archives of Medical Research, vol. 48, no. 8, Article ID S0188440917302357, pp. 766-773, 2017.

[75] M. Kitada, S. Kume, N. Imaizumi, and D. Koya, "Resveratrol improves oxidative stress and protects against diabetic nephropathy through normalization of Mn-SOD dysfunction in AMPK/SIRT1- independent pathway," Diabetes, vol. 60, no. 2, pp. 634-643, 2011.

[76] G. Yildiz, Y. Yildiz, P. A. Ulutas, A. Yaylali, and M. Ural, "Resveratrol pretreatment ameliorates TNBS colitis in rats," Recent Patents on Endocrine Metabolic \& Immune Drug Discovery, vol. 9, no. 2, 2015.

[77] J. L. Barger, T. Kayo, J. M. Vann et al., "Correction: a low dose of dietary resveratrol partially mimics caloric restriction and retards aging parameters in mice," PLoS ONE, vol. 3, no. 6, Article ID e2264, 2008.

[78] Y. Shi, J. Zhou, B. Jiang, and M. Miao, "Resveratrol and inflammatory bowel disease," Annals of the New York Academy of Sciences, vol. 1403, no. 1, pp. 38-47, 2017.
[79] D. L. L. Ca and I. Villegas, "Resveratrol as an anti-inflammatory and anti-aging agent: mechanisms and clinical implications," Molecular Nutrition \& Food Research, vol. 49, no. 5, 2010.

[80] J. Yang, C. Zhu, J. Ye et al., "Resveratrol protects porcine intestinal epithelial cells from deoxynivalenol induced damage via the Nrf2 signaling pathway," Journal of Agricultural and Food Chemistry, 2018.

[81] J. Yao, J.-Y. Wang, L. Liu et al., "Anti-oxidant effects of resveratrol on mice with DSS-induced ulcerative colitis," Archives of Medical Research, vol. 41, no. 4, pp. 288-294, 2010.

[82] A. Mattarei, M. Carraro, M. Azzolini, C. Paradisi, M. Zoratti, and L. Biasutto, "New water-soluble carbamate ester derivatives of resveratrol," Molecules, vol. 19, no. 10, pp. 15900-15917, 2014.

[83] I. M. Kapetanovic, M. Muzzio, Z. Huang, T. N. Thompson, and D. L. McCormick, "Pharmacokinetics, oral bioavailability, and metabolic profile of resveratrol and its dimethylether analog, pterostilbene, in rats," Cancer Chemotherapy and Pharmacology, vol. 68, no. 3, pp. 593-601, 2011.

[84] M. Larrosa, M. J. Yañéz-Gascón, M. V. Selma et al., "Effect of a low dose of dietary resveratrol on colon microbiota, inflammation and tissue damage in a DSS-induced colitis rat mode," Journal of Agricultural and Food Chemistry, vol. 57, no. 6, pp. 2211-2220, 2009.

[85] M. Rotches-Ribalta, M. Urpi-Sarda, R. Llorach et al., "Gut and microbial resveratrol metabolite profiling after moderate long-term consumption of red wine versus dealcoholized red wine in humans by an optimized ultra-high-pressure liquid chromatography tandem mass spectrometry method," Journal of Chromatography A, vol. 1265, pp. 105-113, 2012.

[86] H. Colom, I. Alfaras, M. Maijó, M. E. Juan, and J. M. Planas, "Population pharmacokinetic modeling of trans-resveratrol and its glucuronide and sulfate conjugates after oral and intravenous administration in rats," Pharmaceutical Research, vol. 28, no. 7, pp. 1606-1621, 2011.

[87] M. Muzzio, Z. Huang, S. C. Hu, W. D. Johnson, D. L. Mccormick, and I. M. Kapetanovic, "Determination of resveratrol and its sulfate and glucuronide metabolites in plasma by LC-MS/MS and their pharmacokinetics in dogs," Journal of Pharmaceutical Biomedical Analysis, vol. 59, no. 59, pp. 201-208, 2012.

[88] G. E. S. Faust, D. J. Boocock, K. R. Patel et al., "Oral administration of resveratrol in humans: Evaluation of plasma and urine levels," Cancer Research, vol. 65, 2005.

[89] F. Cardona, C. Andrés-Lacueva, S. Tulipani, F. J. Tinahones, and M. I. Queipo-Ortuño, "Benefits of polyphenols on gut microbiota and implications in human health," The Journal of Nutritional Biochemistry, vol. 24, no. 8, pp. 1415-1422, 2013.

[90] U. Etxeberria, N. Arias, N. Boqué et al., "Reshaping faecal gut microbiota composition by the intake of trans-resveratrol and quercetin in high-fat sucrose diet-fed rats," The Journal of Nutritional Biochemistry, vol. 26, no. 6, pp. 651-660, 2015.

[91] C. Yang, Q. Deng, J. Xu et al., "Sinapic acid and resveratrol alleviate oxidative stress with modulation of gut microbiota in high-fat diet-fed rats," Food Research International, vol. 116, pp. 1202-1211, 2019.

[92] M. Li, Y. Wu, Y. Hu, L. Zhao, and C. Zhang, "Initial gut microbiota structure affects sensitivity to DSS-induced colitis in a mouse model," Science China Life Sciences, pp. 1-8, 2017.

[93] M.-L. Chen, L. Yi, Y. Zhang et al., "Resveratrol attenuates trimethylamine- $\mathrm{N}$-oxide (TMAO)-induced atherosclerosis by regulating TMAO synthesis and bile acid metabolism via 
remodeling of the gut microbiota," MBio, vol. 7, no. 2, pp. 0221002215, 2016.

[94] W. Fang, Effect of High Fat Diet and Oxidated Protein on Small Mice Gut Flora and Redox State, JiangNan University, China, 2012.

[95] P. del Valle, M. R. García-Armesto, D. de Arriaga, C. GonzálezDonquiles, P. Rodríquez-Fernández, and J. Rúa, "Antimicrobial activity of kaempferol and resveratrol in binary combinations with parabens or propyl gallate against Enterococcus faecalis," Food Control, vol. 61, pp. 213-220, 2016.

[96] A. S. Wellman, M. R. Metukuri, N. Kazgan et al., "Intestinal epithelial sirtuin 1 regulates intestinal inflammation during aging in mice by altering the intestinal microbiota," Gastroenterology, vol. 153, no. 3, pp. 772-786, 2017.

[97] M. Lagouge, C. Argmann, Z. Gerhart-Hines et al., "Resveratrol improves mitochondrial function and protects against metabolicdisease by activating SIRT1 and PGC-1 $\alpha$," Cell, vol. 127, no. 6, pp. 1109-1122, 2006.

[98] N. L. Price, A. P. Gomes, A. J. Y. Ling et al., "SIRT1 is required for AMPK activation and the beneficial effects of resveratrol on mitochondrial function," Cell Metabolism, vol. 15, no. 5, pp. 675690, 2012.

[99] G. Torres Santiago, J. I. Serrano Contreras, M. E. Meléndez Camargo, and L. G. Zepeda Vallejo, "NMR-based metabonomic approach reveals changes in the urinary and fecal metabolome caused by resveratrol," Journal of Pharmaceutical and Biomedical Analysis, vol. 162, pp. 234-241, 2019.

[100] L. M. Bode, D. Bunzel, M. Huch et al., "In vivo and in vitro metabolism of trans-resveratrol by human gut microbiota," American Journal of Clinical Nutrition, vol. 97, no. 2, pp. 295309, 2013.

[101] C. M. Jung, T. M. Heinze, L. K. Schnackenberg et al., "Interaction of dietary resveratrol with animal-associated bacteria," FEMS Microbiology Letters, vol. 297, no. 2, pp. 266-273, 2009.

[102] D. Lin, F. Dai, L.-D. Sun, and B. Zhou, "Toward an understanding of the role of a catechol moiety in cancer chemoprevention: the case of copper- and o-quinone-dependent Nrf2 activation by a catechol-type resveratrol analog," Molecular Nutrition \& Food Research, vol. 59, no. 12, pp. 2395-2406, 2015.

[103] S. W. Tsang, Y.-F. Guan, J. Wang, Z.-X. Bian, and H.-J. Zhang, "Inhibition of pancreatic oxidative damage by stilbene derivative dihydro-resveratrol: implication for treatment of acute pancreatitis," Scientific Reports, vol. 6, no. 22859, Article ID 22859, 2016.

[104] S. Fabris, F. Momo, G. Ravagnan, and R. Stevanato, "Antioxidant properties of resveratrol and piceid on lipid peroxidation in micelles and monolamellar liposomes," Biophysical Chemistry, vol. 135, no. 1-3, pp. 76-83, 2008.

[105] Y. Chen, D.-Q. Zhang, Z. Liao et al., "Anti-oxidant polydatin (piceid) protects against substantia nigral motor degeneration in multiple rodent models of Parkinson's disease," Molecular Neurodegeneration, vol. 10, no. 1, 2015.

[106] K. Driscoll, A. Deshpande, A. Chapp, K. Li, R. Datta, and W. Ramakrishna, "Anti-inflammatory and immune-modulating effects of rice callus suspension culture (RCSC) and bioactive fractions in an in vitro inflammatory bowel disease model," Phytomedicine, vol. 57, pp. 364-376, 2019.

[107] Y. Zhu, W.-H. Pan, C. F. Ku, H.-J. Zhang, and S. W. Tsang, "Design, synthesis and evaluation of novel dihydrostilbene derivatives as potential anti-melanogenic skin-protecting agents," European Journal of Medicinal Chemistry, vol. 143, pp. 1254-1260, 2018.
[108] Z.-S. Lin, C. F. Ku, Y.-F. Guan et al., "Dihydro-resveratrol ameliorates lung injury in rats with cerulein-induced acute pancreatitis," Phytotherapy Research, vol. 30, no. 4, pp. 663-670, 2016.

[109] T. T. Kim, N. Parajuli, M. M. Sung et al., "Fecal transplant from resveratrol-fed donors improves glycaemia and cardiovascular features of the metabolic syndrome in mice," American Journal of Physiology-Endocrinology and Metabolism, vol. 315, no. 4, pp. E511-E519, 2018.

[110] M. M. Sung, T. T. Kim, E. Denou et al., "Improved glucose homeostasis in obese mice treated with resveratrol is associated with alterations in the gut microbiome," Diabetes, vol. 66, no. 2, pp. 418-425, 2017. 


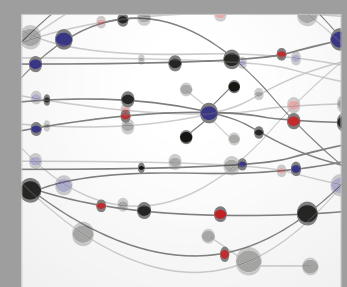

The Scientific World Journal
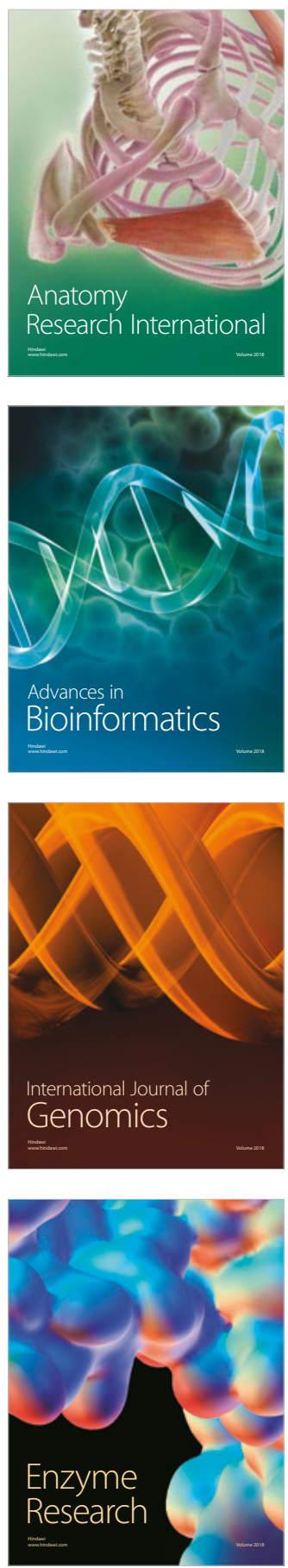
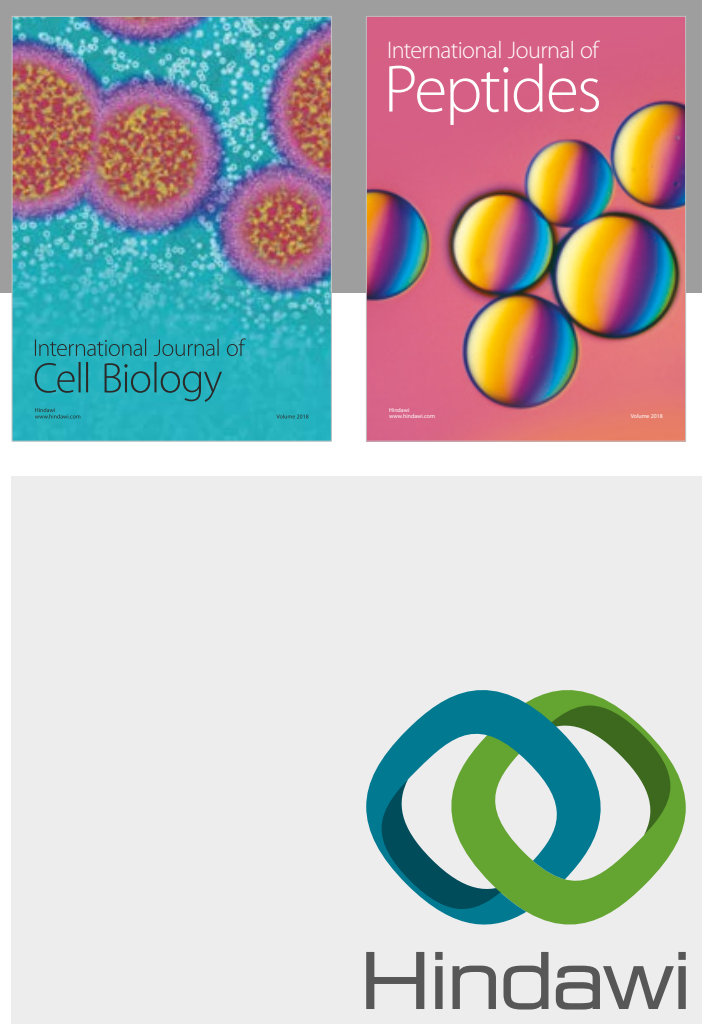

Submit your manuscripts at

www.hindawi.com
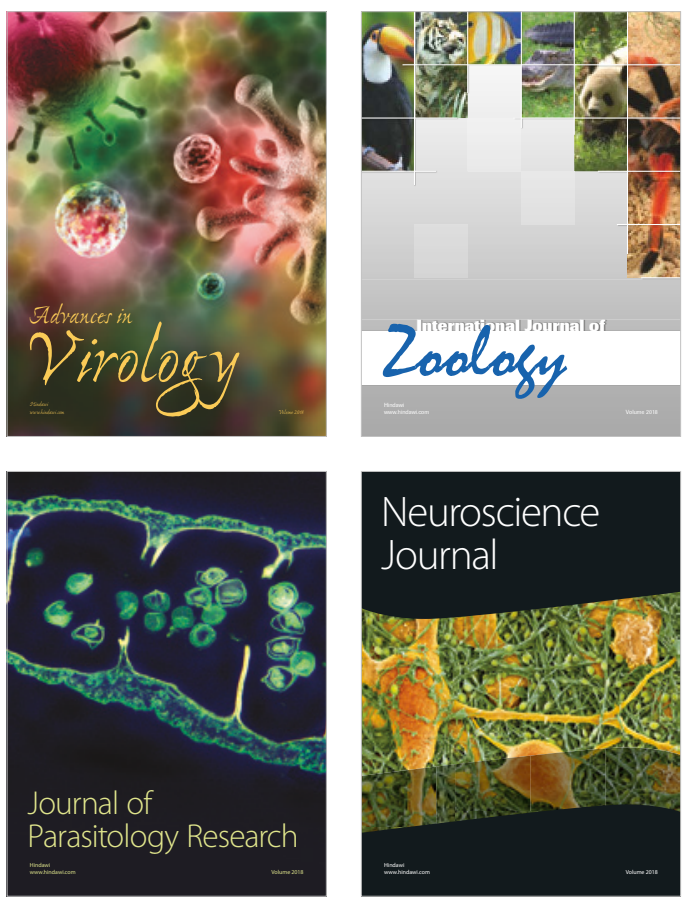
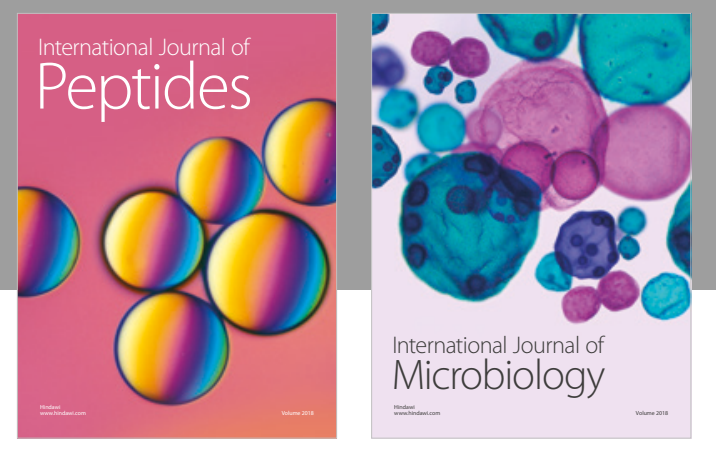

nternational Journal of Microbiology
Journal of
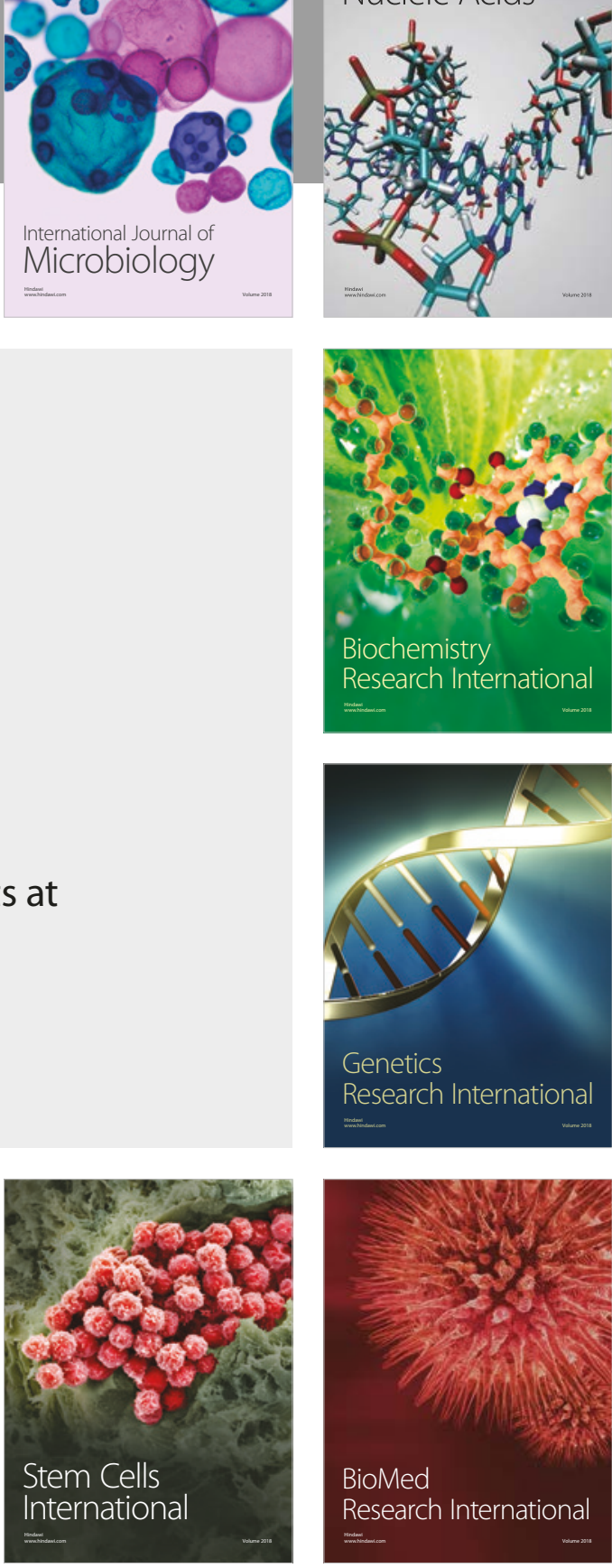
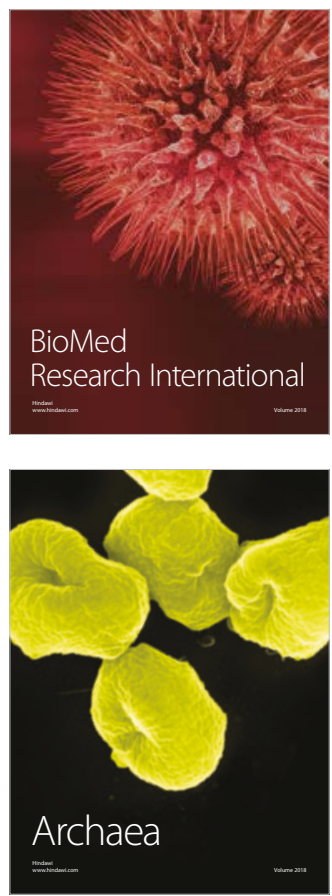